\title{
Diversidade de borboletas (Lepidoptera, Papilionoidea e Hesperioidea) em fragmentos de Floresta Estacional Decidual em Santa Maria, Rio Grande do Sul, Brasil
}

\author{
Mônica B. Dessuy \& Ana B. B. de Morais
}

Programa de Pós-Graduação em Biodiversidade Animal, Departamento de Biologia, Centro de Ciências Naturais e Exatas, Universidade Federal de Santa Maria. Faixa de Camobi, km 09, 97105-900 Santa Maria, Rio Grande do Sul, Brasil. E-mail: monicadessuy@yahoo.com.br

\begin{abstract}
Diversity of butterflies (Lepidoptera, Papilionoidea e Hesperioidea) in fragments of decidual seasonal forest in Santa Maria, Rio Grande do Sul State, Brazil. Six field trips were carried bimonthly, between July 2004 and July 2005, in three fragments of Decidual Seasonal Forest of Santa Maria, Rio Grande do Sul State: Morro do Elefante (E), Morro do Lar Metodista (M) and Morro Cerrito (C). After 135 sampling hours, 1594 butterflies of 145 species were registered. Among these, 59\% are Nymphalidae, 19\% Hesperiidae, 10\% Papilionidae, 7\% Pieridae and 5\% Lycaenidae. Nine species were new registers for Rio Grande do Sul State. $M$ had the highest richness and abundance of species. The lowest richness was observed in $\mathrm{E}$ and the lowest abundance in $\mathrm{C}$. Shannon-Wiener and Margalef diversity indexes had the same ordination among localities, being higher in $\mathrm{M}$, more heterogeneous and disturbed, and lower in E. Simpson and Berger-Parker dominance indexes were higher in $\mathrm{E}$, with more abundant species, and lower in C. Only $30 \%$ of the species were common to the three localities. About half of the total registered species were exclusive from one of the three localities. The highest similarity indexes (Morisita and Jaccard) were obtained between $\mathrm{M}$ and $\mathrm{E}$, and the lowest between $\mathrm{E}$ and $\mathrm{C}$.

KEY WORDS. Butterfly; conservation; inventory; South Brazil; species richness.
\end{abstract}

RESUMO. Foram realizadas seis saídas a campo bimestrais, entre julho de 2004 e julho de 2005, em três fragmentos de Floresta Estacional Decidual de Santa Maria, Rio Grande do Sul: Morro do Elefante (E), Morro do Lar Metodista (M) e Morro Cerrito (C). Em 135 horas de amostragem, foram registradas 1594 borboletas, distribuídas em 145 espécies. Destas, 59\% pertencem a família Nymphalidae, 19\% Hesperiidae, 10\% Papilionidae, 7\% Pieridae e $5 \%$ Lycaenidae. Foram registradas nove espécies de borboletas ainda não publicadas para o Estado. M apresentou maior riqueza e abundância de espécies. A menor riqueza foi observada em $E$ e a menor abundância em $C$. Os índices de diversidade de Shannon-Wiener e de Margalef tiveram a mesma ordenação entre os locais, sendo maiores em $\mathrm{M}$, local mais heterogêneo e perturbado, e menores em E. Os índices de dominância de Simpson e de Berger-Parker, por sua vez, foram mais representativos em $\mathrm{E}$, com o maior número de espécies abundantes, e menos em C. Apenas 30\% das espécies foram comuns aos três locais. Cerca da metade do total de espécies registradas foram exclusivas de um dos locais. A maior similaridade (Índices de Morisita e de Jaccard) foi observada entre M e $E$, e a menor entre $E$ e $C$.

PALAVRAS-CHAVE. Borboletas; conservação; inventário; riqueza de espécies, sul do Brasil.

Os lepidópteros formam uma das mais abundantes ordens de insetos, com cerca de 146000 espécies descritas (HEPPNER 1991). As borboletas possuem hábito diurno e são representadas por cinco famílias, Hesperiidae, Papilionidae, Pieridae, Lycaenidae e Nymphalidae. Na região Neotropical, as borboletas somam entre 7100 (Beccalonı \& Gaston 1995) e 7900 espécies (Heppner 1991) enquanto no Brasil ocorrem entre 3100 (BECCALONI \& GASTON 1995) e 3200 espécies (Brown JR \& Freitas 1999).
As borboletas estão envolvidas em muitas interações ecológicas dentro das comunidades a que pertencem, destacandose as mutualísticas (polinização) e de predação (herbivoria), dentre outras. Elas ainda servem como modelo em pesquisas de ecologia de populações e comportamento (pela facilidade de marcação nas asas); genética da seleção natural e em processos básicos como: alimentação, parasitismo, competição e predação (identificação de substâncias tóxicas presentes, camuflagem e 
mimetismo) (Boggs et al. 2003). No Brasil, são objeto de muitos estudos científicos (BRown JR 1996), podendo ainda ser usadas como indicadoras em levantamentos de fauna e determinação de prioridades, planejamento e administração de reservas naturais (Brown JR 1992, Brown JR \& FreitAs 2002, Emery et al. 2006).

A diversidade de borboletas (mais do que a riqueza) está significativamente correlacionada tanto com a área de mata como com seu grau de isolamento (BAz \& BOYERo 1995). As diferenças na diversidade entre habitats podem ser atribuídas à variedade de condições apropriadas para as borboletas, o que inclui fonte de néctar, água, lama e incidência de luz solar (Brown JR \& Hutchings 1997, Tumuhimbise et al. 2001), além da conectividade entreáreas de mata ejardins cultivados (BRown JR \& FrEITAS 2002). Devido ao fato de possuírem representantes com tamanho grande, coloridos e de fácil visualização, as borboletas poderiam ser usadas como "bandeiras" para conservação e indicadores para monitoramento ambiental (New 1997). Dentre os motivos para tais usos, destaca-se o fato de serem comuns o ano inteiro, apresentarem grande diversidade, facilidade de amostragem e identificação, ciclos de vida pequenos e facilidade de criação em laboratório. Ainda respondem rapidamente a alterações ambientais, por serem especialistas em recursos específicos e possuírem fidelidade de microhabitat, permitindo assim ações rápidas como reação a degradação do habitat. Sua presença pode indicar uma continuidade de sistemas frágeis e comunidades ricas em espécies, e sua ausência uma perturbação, fragmentação ou envenenamento forte demais para manter a integridade dos sistemas e da paisagem (New et al. 1995, Brown JR 1996, Brown JR \& Hutchings 1997, Brown JR \& Freitas 1999).

No Estado do Rio Grande do Sul, não existe um registro completo das espécies de borboletas que ocorrem. A maioria dos trabalhos disponíveis foi executada com objetivos diversos, utilizando metodologias distintas ou não explicitadas. O projeto "As Borboletas do Rio Grande do Sul", desenvolvido no Departamento de Zoologia da UFRGS desde 1996, vem estudando a fauna de borboletas das Unidades de Conservação do Estado, utilizando métodos padronizados (ISERHARD \& ROMANOWSKI 2004), sem, no entanto, ter amostrado a região central. O presente estudo, utilizando a metodologia do referido projeto, busca caracterizar a fauna de borboletas de fragmentos da Floresta Estacional Decidual da Fralda da Serra Geral de Santa Maria, através de listagem de espécies e medidas de riqueza, abundância, diversidade, dominância e similaridade. Pretende-se fornecer ainda subsídios para conservação desse grupo de insetos e do ambiente natural da região, carente de áreas legais de preservação, e atualmente sob forte pressão antrópica.

\section{MATERIAL E MÉTODOS}

\section{Área de Estudo}

Localizado no centro geográfico do Rio Grande do Sul, o município de Santa Maria (2942'S e 5342'W) (Fig. 1) possui clima temperado chuvoso e quente, do tipo $\mathrm{Cfa}$, com nevoeiros. A precipitação média anual é de 1732 mm e a temperatura

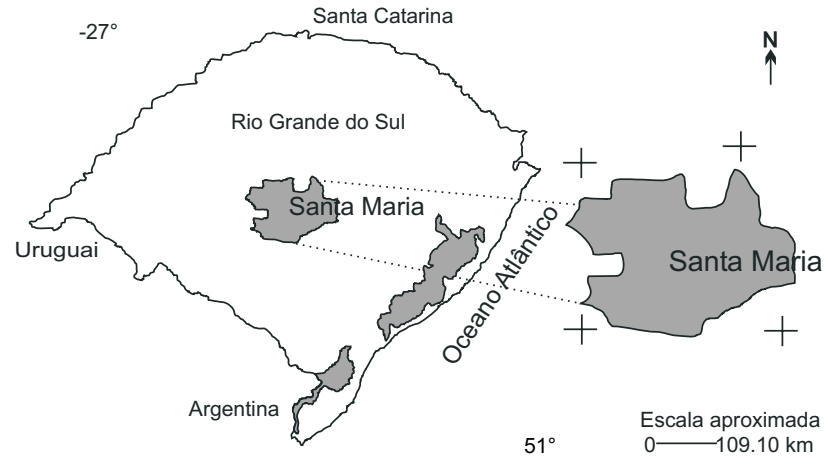

Figura 1. Localização do município de Santa Maria (2942'S, 53은'W), Rio Grande do Sul, Brasil.

média anual oscila entre 18ㅇ C e 20 C (NIMER 1990). Em termos de vegetação, pertence à Floresta Estacional Decidual da Fral da da Serra Geral (Pereira et al. 1989), ocupando a zona de transição entre a Depressão Central do Rio Grande do Sul e a escarpa arenito-basáltica do Planalto Meridional Brasileiro, possuindo planícies aluviais, várzeas e coxilhas, com altitudes variando de 40 a 500 metros. Originalmente cobrindo os morros da cidade com vegetação de espécies características de matas, a Floresta Estacional Decidual da Fralda da Serra Geral atualmente concentra-se em locais de difícil acesso, em decorrência do intenso desmatamento para ocupação residencial, expansão da área agrícola e das explorações das madeireiras (Pereira et al. 1989, Robaina et al. 2001).

Foram escolhidos três locais para a realização desta pesquisa: Morro do Elefante (2940'57"S e 5343'17"W), Morro do Lar Metodista (2939'38"S e 5349'8"W) e Morro Cerrito (2941'59"S e 5347'18"W). Os Morros do Elefante (E) e do Lar Metodista (M) localizam-se na escarpa do Planalto Médio Riograndense ou da Fralda da Serra Geral, a 9,5 km de distância um do outro, e possuem altitudes entre 420 e 470 m (MACHADO \& LONGHI 1990). O Morro Cerrito (C), isolado e já na Depressão Central, é tido como testemunha da Serra Geral e possui altitude de $246 \mathrm{~m}$ (Pereira et al. 1989), estando a $5 \mathrm{~km}$ de E e $7 \mathrm{~km}$ de $\mathrm{C}$. A escolha desses fragmentos foi devido ao fato de serem típicos e característicos da região, cobertos por matas naturais do tipo Estacional Decidual, ainda razoavelmente preservadas pelo fato das áreas estarem sob a responsabilidade de instituições privadas.

Na vegetação do extrato arbóreo dos morros ao redor da cidade de Santa Maria, as famílias botânicas mais presentes são Fabaceae e Myrtaceae, seguidas de Lauraceae, Meliaceae, Sapindaceae, Euphorbiaceae e Moraceae. Predominam os gê neros Trichilia, Eugenia, Nectandra, Allophylus, Rapanea e Zanthoxylum. Embora apresentem grande semel hança florística, principalmente com relação às famílias botânicas ocorrentes, a composição de espécies ganha aspectos peculiares a cada local (Machado \& Longhi 1990, Alberti et al. 2000).

Revista Brasileira de Zoologia 24 (1): 108-120, março 2007 
O Morro do Elefante localiza-se atrás do Educandário conhecido como "Cidade dos Meninos", no bairro Camobi, e possui área de aproximadamente 200 ha. Dentre a vegetação natural, existem algumas áreas plantadas com eucalipto, Pinus e frutíferas cultivadas (principalmente Citrus). Na entrada da trilha principal da mata existe um trecho de gramado aberto ensolarado, ao lado do qual corre um riacho proveniente do interior da mata. O Morro do Lar Metodista, por sua vez, localiza-se atrás do Lar M etodista, no bairro Chácara das Flores, e possui área intermediária entre os outros dois locais. Dos três locais, esse é o mais antropizado, com presença de clareiras bem abertas no interior dos trechos de mata, alternando-se com trechos de campo e capoeira. Ao longo da entrada da área, na base do morro, estende-se um riacho. Já o Morro Cerrito pertence ao Centro Marista de Eventos, localizado no bairro também conhecido como Cerrito, e possui área de aproximadamente 20 hectares. Possui alguns trechos com presença de eucalipto, taquara e Pinus, e ainda um pomar com várias frutíferas cultivadas. Logo na entrada da mata, há uma grande clareira, devido à construção de uma quadra de esportes, e ainda a presença de gramados e jardins cultivados desde a entrada da sede administrativa até a trilha de acesso a mata.

\section{Amostragem}

Em cada local foram realizadas amostragens bimestrais, de julho de 2004 a julho de 2005, percorrendo-se todos os tipos de ambiente característicos, incluindo interior e borda de mata. O esforço amostral foi de cerca de 3,5 horas-rede/ local/ ocasião, ao longo de trilhas já existentes nos locais, que eram percorridas uma única vez em cada amostragem, baseado na metodologia proposta por PolLARD (1977). O horário de amostragem estendeu-se entre 8h30min e $16 \mathrm{~h}$, procurando acompanhar o horário de maior atividade das borboletas.

As borboletas, visualizadas a olho nu ou com binóculo, eram registradas em planilha e/ou capturadas com rede entomológica, caso não fosse possível a identificação no campo. Os exemplares capturados eram mortos por compressão do tórax e acondicionados em envelope entomológico, a fim de serem levados ao laboratório para montagem. A identificação dos espécimes foi realizada com auxílio de bibliografia especializada (D'Abrera 1988, Brown J R 1992, Tyler et al. 1994, D'ABrera 1994, 1995, CANALS 2000, 2003) e consulta a coleções de outras instituições e/ou especialistas. Pelo menos um exemplar de cada espécie está depositado na coleção de referência do Laboratório de Interações Inseto-Planta do Departamento de Biologia, Centro de Ciências Naturais e Exatas da Universidade Federal de Santa Maria, Santa Maria, Rio Grande do Sul. Também foram confeccionadas coleções menores, específicas e representativas de cada local, para serem depositadas nas sedes administrativas dos mesmos.

\section{Análise dos Dados}

Foram analisados os seguintes itens: número de indivíduos registrados $(N)$, riqueza de espécies $(S)$, freqüência relati- va da espécie (fr) e número de espécies exclusivas (Excl.). Para anál ise de abundância das espécies, foram consideradas "abundantes" aquelas que possuíam as mais altas freqüências absolutas e como "dominantes" as que apresentaram freqüência relativa maior que $10 \%$ ( $\mathrm{fr}>0,1$ ). Espécies registradas em apenas um dos três locais são referidas como "exclusivas". Espécies representadas por um único indivíduo foram referidas como “singletons" (Novotný \& BASSET 2000).

Foi feita uma curva de suficiência amostral, a partir dos dados de número de espécies obtidas em cada ocasião amostral, utilizando-se o programa estatístico Estimates (CoLwELL 2005).

Também foram calculados os Índices de Diversidade de Shannon-Wiener $\left(\mathrm{H}^{\prime}\right)$ e de Margalef (Dmg), Índices de Dominância de Simpson (D) e de Berger-Parker (d) e Índices de Similaridade de Morisita-Horn e de Jaccard (Moreno 2001, MAGURRAN 2004), utilizando o programa estatístico Past, versão 1,35b.

A elaboração da listagem de espécies seguiu a classificação de Brown JR (1992) e FrEITAS \& BROWn JR (2004) e a nomenclatura foi atualizada de acordo com LAMAS (2004) e MieLKE (2005).

\section{RESULTADOS E DISCUSSÃO}

\section{Riqueza e Composição de Espécies}

Em 135 horas de amostragem, foram registrados 1564 indivíduos, distribuídos em 145 espécies de borboletas (Tab. I). Destes, 925 (59\%) pertencem à família Nymphalidae; 299 (19\%) são Hesperiidae; 158 (10\%) Papilionidae; 97 (7\%) Pieridae e 85 (5\%) Lycaenidae.

Do total de 145 espécies identificadas, 40\% são Hesperiidae, 35\% Nymphalidae, 11\% Lycaenidae, 8\% Papilionidae e 6\% Pieridae, proporções diferentes das apresentadas por BRown JR \& FreitAs (1999), que citam Lycaenidae e Hesperiidae como as famílias mais ricas em espécies no país, seguidas de Nymphalidae, Pieridae e Papilionidae (Tab. II). Trabalhos mais regionais encontraram pequenas diferenças na representatividade da riqueza de espécies por família com relação aos dados apresentados para o Brasil (MIelke \& CASAgrande 1997 e Brown JR \& Freitas 1999 em São Paulo, Motta 2002 em Minas Gerais, Krüger \& Si lva 2003 no Rio Grande do Sul, Emery et al. 2006 no Distrito Federal, com exceção de Hesperiidae nos dois últimos). No entanto, no Rio Grande do Sul, ISERHARD \& RomANOWSKI (2004), na região do Vale do Rio Maquiné, encontraram valores mais próximos aos do presente estudo, sendo Nymphalidae a família mais rica, seguida por Hesperiidae, Lycaenidae, Pieridae e Papilionidae (Tab. II). Assim, apresenta-se um padrão para o Estado que aponta menor riqueza de Lycaenidae em relação ao Brasil, citada como aproximadamente 30\% (Brown JR \& FreitAs 1999), enfatizando a necessidade de ampliar esse tipo de estudo no sul do país (MIelKe \& CASAgrande 1997).

Cinqüenta e oito espécies de Hesperiidae foram registradas no presente trabal ho e duas constituem-se no primeiro registro publicado, até o momento, para o Estado: Cymaenes distigma Plötz, 1883 (Morro do Elefante, 22.II.2005, Mielke det.; 
Tabela I. Lista e abundância de espécies de borboletas registradas no Morro do Elefante (E), Morro do Lar Metodista (M) e Morro Cerrito (C), Santa Maria, Rio Grande do Sul, entre julho de 2004 e julho de 2005. (T) Total das amostragens; (S) riqueza de espécies; (N) abundância total por família em cada local; em negrito: espécies com novo registro para o Rio Grande do Sul; (*) espécies representadas por um único indivíduo ("singletons") e consideradas raras ou escassas na literatura (Biezanko 1963, BiezAnko \& MiekE 1973, Biezanko et al. 1978, Brown JR 1992, Mielke \& Casagrande 1997).

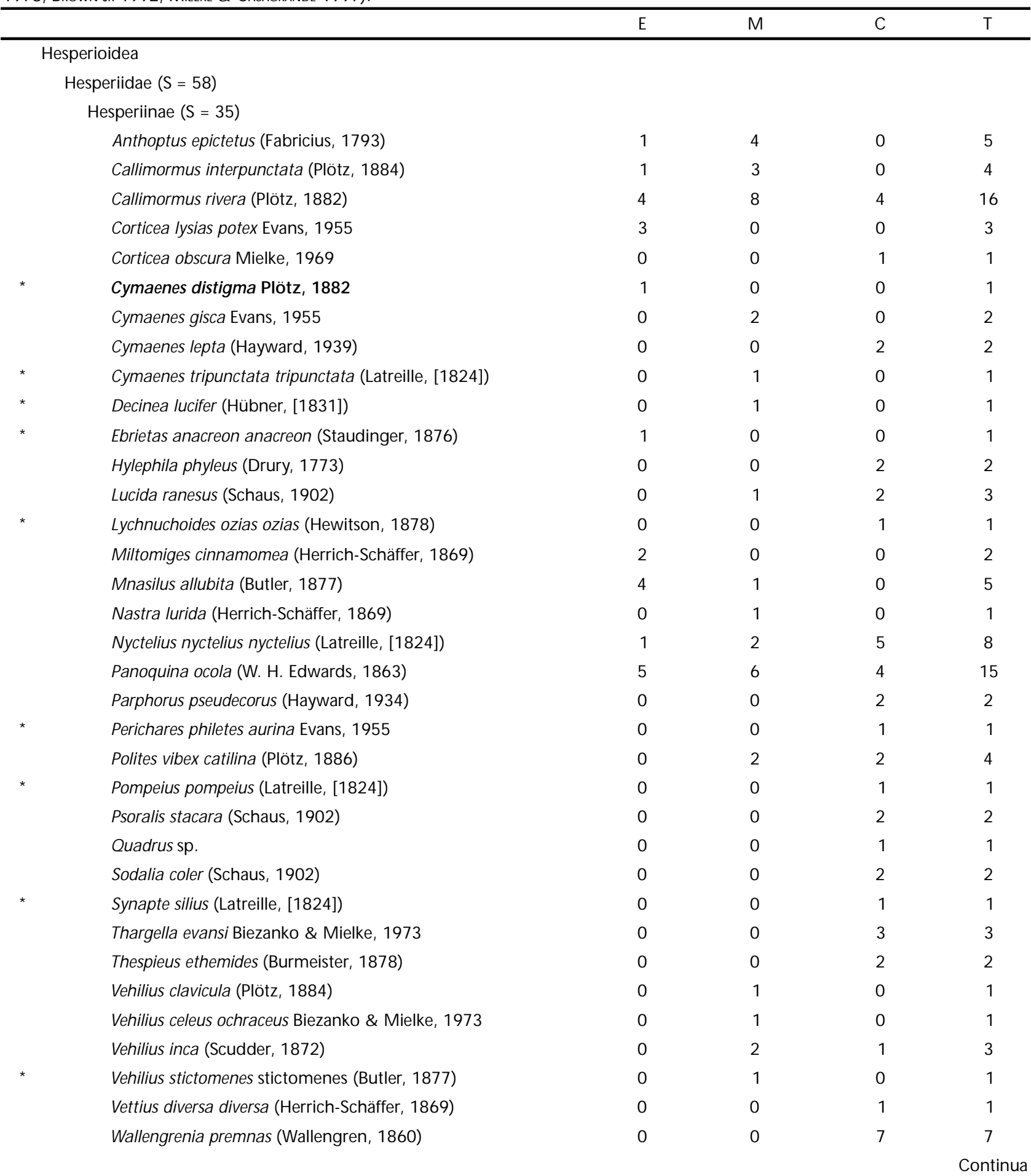


Tabela I. Continuação.

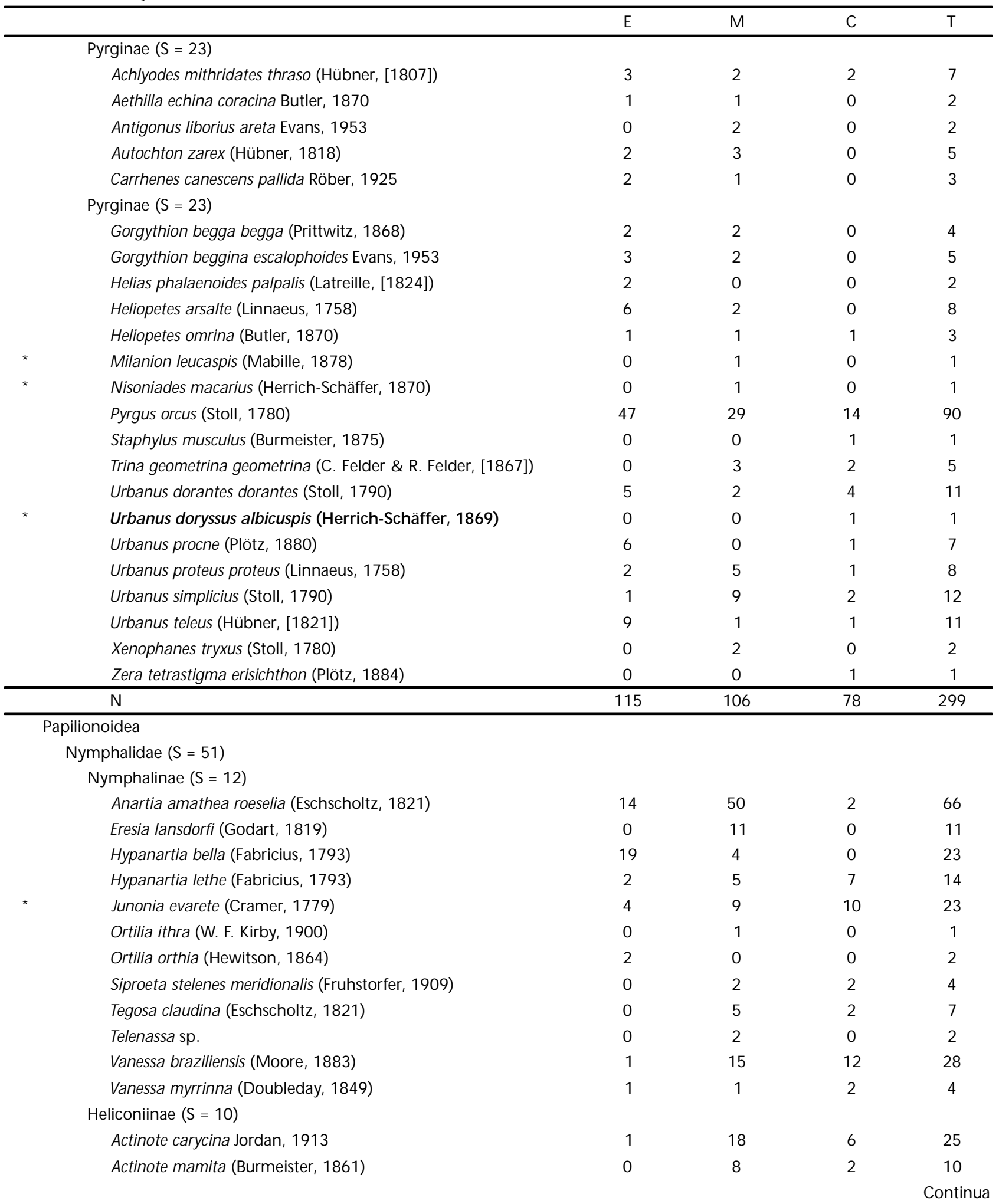

Revista Brasileira de Zoologia 24 (1): 108-120, março 2007 
Tabela I. Continuação.

\begin{tabular}{|c|c|c|c|c|c|}
\hline & & $\mathrm{E}$ & M & $\mathrm{C}$ & $\mathrm{T}$ \\
\hline & Actinote melanisans Oberthür, 1917 & 2 & 3 & 0 & 5 \\
\hline & Actinote pellenea calymma Jordan, 1913 & 0 & 2 & 0 & 2 \\
\hline & Actinote thalia pyrrha (Fabricius, 1775) & 3 & 3 & 0 & 6 \\
\hline & Agraulis vanillae maculosa (Stichel, [1908]) & 0 & 3 & 2 & 5 \\
\hline & Dione juno juno (Cramer, 1779) & 0 & 0 & 7 & 7 \\
\hline & Dryas iulia alcionea (Cramer, 1779) & 52 & 58 & 36 & 146 \\
\hline & Heliconius erato phyllis (Fabricius, 1775) & 55 & 43 & 23 & 121 \\
\hline & Heliconius ethilla narcaea Godart, 1819 & 7 & 5 & 11 & 23 \\
\hline & \multicolumn{5}{|l|}{ Satyrinae $(S=9)$} \\
\hline & Capronnieria galesus (Godart, [1824]) & 0 & 1 & 0 & 1 \\
\hline & Hermeuptychia hermes (Fabricius, 1775) & 19 & 18 & 12 & 49 \\
\hline & Moneuptychia paeon (Godart, [1824]) & 1 & 1 & 0 & 2 \\
\hline & Moneuptychia soter (Butler, 1877) & 1 & 3 & 1 & 5 \\
\hline & Paryphthimoides phronius (Godart, [1824]) & 41 & 19 & 30 & 90 \\
\hline & Paryphthimoides poltys (Prittwitz, 1865) & 6 & 13 & 3 & 22 \\
\hline & Praepedaliodes phanias (Hewitson, 1862) & 0 & 0 & 1 & 1 \\
\hline & Pseudodebis euptychidia (Butler, 1868) & 7 & 0 & 1 & 8 \\
\hline & Yphthimoides celmis (Godart, [1824]) & 1 & 14 & 22 & 37 \\
\hline & \multicolumn{5}{|l|}{ Ithomiinae $(S=7)$} \\
\hline & Dircenna dero (Hübner, 1823) & 0 & 1 & 0 & 1 \\
\hline & Epityches eupompe (Geyer, 1832) & 3 & 8 & 0 & 11 \\
\hline & Episcada hymenaea hymenaea (Prittwitz, 1865) & 1 & 3 & 0 & 4 \\
\hline & Mechanitis lysimnia lysimnia (Fabricius, 1793) & 8 & 4 & 1 & 13 \\
\hline & Methona themisto (Hübner, 1818) & 2 & 1 & 1 & 4 \\
\hline \multirow[t]{5}{*}{$*$} & Pseudoscada erruca (Hewitson, 1855) & 2 & 3 & 0 & 5 \\
\hline & Pteronymia sylvo (Geyer, 1832) & 0 & 1 & 0 & 1 \\
\hline & \multicolumn{5}{|l|}{ Biblidinae $(S=6)$} \\
\hline & Biblis hyperia (Cramer, 1779) & 1 & 3 & 3 & 7 \\
\hline & Callicore pygas (Godart, [1824]) & 0 & 0 & 1 & 1 \\
\hline \multirow[t]{17}{*}{ * } & Dynamine myrrhina (Doubleday, 1849) & 1 & 3 & 0 & 4 \\
\hline & Dynamine postverta postverta (Cramer, 1779) & 0 & 0 & 1 & 1 \\
\hline & Eunica eburnea Fruhstorfer, 1907 & 0 & 1 & 1 & 2 \\
\hline & Hamadryas epinome (C. Felder \& R. Felder, 1867) & 28 & 5 & 2 & 35 \\
\hline & \multicolumn{5}{|l|}{ Danainae $(S=2)$} \\
\hline & Danaus gilippus gilippus (Cramer, 1775) & 1 & 11 & 2 & 14 \\
\hline & Danaus erippus (Cramer, 1775) & 5 & 20 & 1 & 26 \\
\hline & \multicolumn{5}{|l|}{ Limenitidinae ( $\mathrm{S}=2$ ) } \\
\hline & Adelpha syma (Godart, [1824]) & 0 & 1 & 0 & 1 \\
\hline & Adelpha thessalia indefecta Fruhstorfer, 1913 & 1 & 0 & 1 & 2 \\
\hline & \multicolumn{5}{|l|}{ Apaturinae $(S=1)$} \\
\hline & Doxocopa laurentia laurentia (Godart, [1824]) & 2 & 0 & 2 & 4 \\
\hline & \multicolumn{5}{|l|}{ Brassolinae $(S=1)$} \\
\hline & Brassolis sophorae vulpeculus Stichel, 1902 & 7 & 2 & 0 & 9 \\
\hline & \multicolumn{5}{|l|}{ Morphinae $(S=2)$} \\
\hline & Morpho aega aega (Hübner, [1822]) & 20 & 1 & 9 & 30 \\
\hline & $\mathrm{N}$ & 321 & 385 & 219 & 925 \\
\hline
\end{tabular}


Tabela I. Continuação.

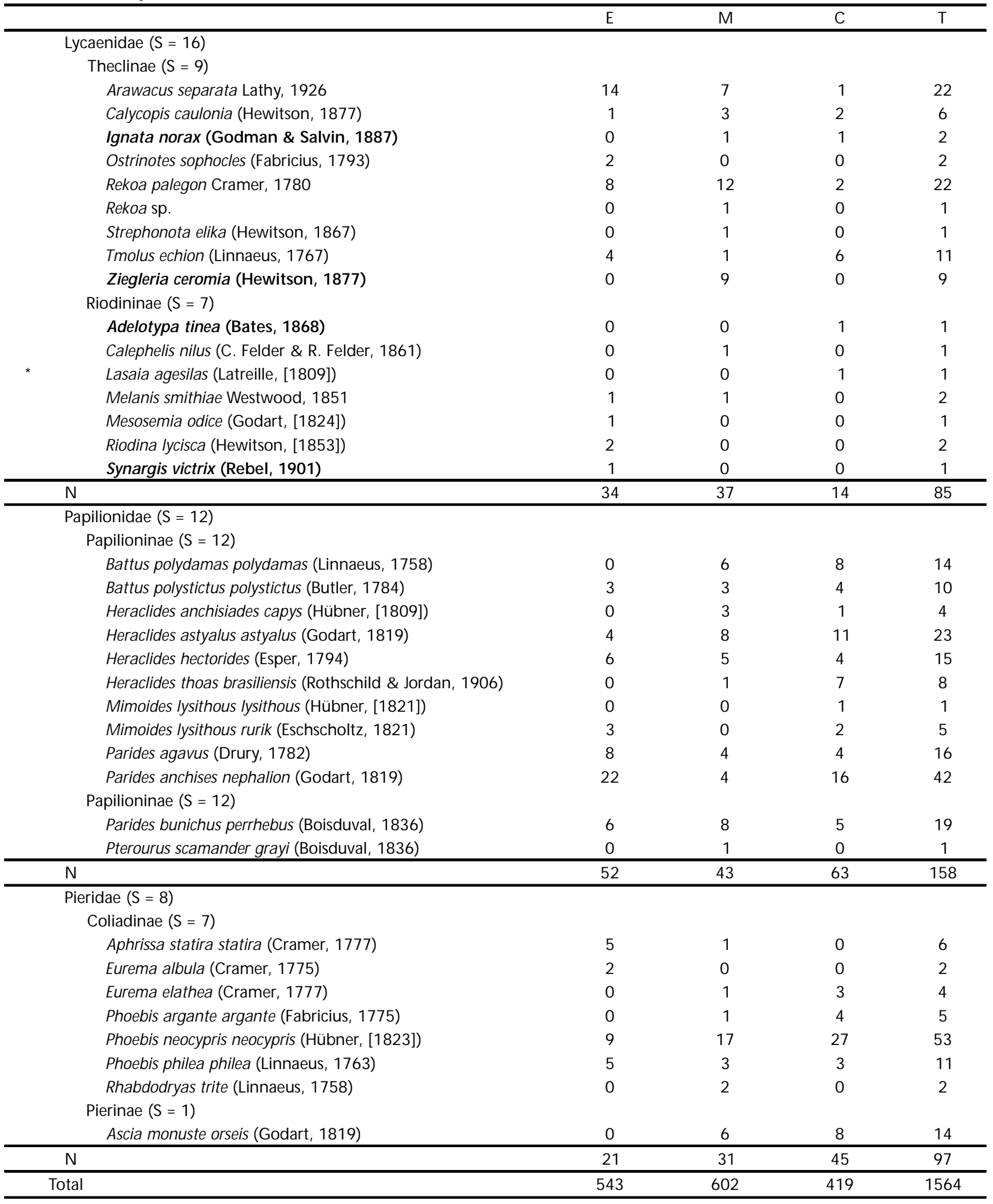

Revista Brasileira de Zoologia 24 (1): 108-120, março 2007 
Tabela II. Riqueza de espécies por família (\%) em comunidades de borboletas no Brasil e no Rio Grande do Sul. (B\&F) Brown \& FretAS (1999); (I\&R) IseRHARD \& RomanowskI (2004), (SM) Santa Maria.

\begin{tabular}{lccccc}
\hline & Brasil & & \multicolumn{2}{c}{ Rio Grande do Sul } \\
\cline { 2 - 2 } \cline { 5 - 5 } \cline { 5 - 5 } & B\&F & & I\&R & SM \\
\hline Hesperiidae & 36 & & 33 & 40 \\
Papilionidae & 2 & & 4 & 8 \\
Pieridae & 2 & & 8 & 6 \\
Lycaenidae & 36 & & 19 & 11 \\
Nymphalidae & 24 & & 36 & 35 \\
\hline
\end{tabular}

Morais leg.) eUrbanus doryssus albicuspis (Herrich-Schäffer, 1869) (Morro Cerrito, 19.X.2004, Dessuy det.; Dessuy leg.) (Biezanko 1963, Biezanko \& Mielke 1973, Mielke 1979, Mielke 2005, MARCHIORI \& ROMANOWSKI 2006).

Das 51 espécies de Nymphalidae encontradas, três foram registradas pela primeira vez no Estado: Callicore pygas (Godart, [1824]) (Morro Cerrito, 17.XII.2004, Dessuy det.; Morais leg.), Dynamine postverta postverta (Cramer, 1779) (Morro Cerrito, 09.XII.2004, Dessuy det.; Dessuy leg.) e Pseudodebis euptychidia (Butler, 1868) (Morro do Elefante, 05.XII.2004, Dessuy det.; Dessuy leg.) (Biezanko \& Freitas 1938, Biezanko 1949, 1960b, c, d, e, Teston \& Corseuil 2001, 2002a, b, Krüger \& Silva 2003, ISERHARD \& ROMANOWSKI 2004, QuADROS et al. 2004, MARCHIORI \& ROMANOWSKI 2006).

Segundo Brown JR \& Freitas (1999) Lycaenidae é quase tão diversificada quanto Nymphalidae e importante como indicadora, assim como Hesperiidae. No entanto, essas borboletas são de difícil amostragem, e existem poucos inventários dessa família no Rio Grande do Sul. No presente trabalho, foram encontradas 16 espécies desta família, quatro pela primeira vez no Estado: Adelotypa tinea (Bates, 1868) (Morro Cerrito, 17.XII.2004, Robbins det.; Morais leg.), Ignata norax (Godman \& Salvin, 1887) (Morro Cerrito, 17.XII.2004, Robbins det.; Dessuy leg.), Synargis victrix (Rebel, 1901) (Morro do Elefante, 14.XII.2004, Dessuy det.; Dessuy leg.) e Ziegleria ceromia (Hewitson, 1877) (Morro do Lar Metodista, 15.XII.2004, Robbins det.; Dessuy leg.) (Biezanko et al. 1978, Krüger \& Silva 2003, Corseuil et al. 2004, Iserhard \& RomanowsKI 2004).

Doze espécies de Papilionidae e oito de Pieridae foram registradas no presente trabalho, nenhuma constituindo-se em registro novo para o Rio Grande do Sul (BIEZANKo \& FreITAS 1938, Biezanko 1958, 1959a, b, 1960a, Teston \& Corseuil 1998, 1999, 2000a, b, KRÜGer \& Silva 2003, IserhaRd \& RomanowsKI 2004). Em Santa Maria, trabalhos anteriores com Pieridae e Papilionidae (Link et al. 1977, SchwarTz \& Dı MARE 2001) efetuados em vários ambientes com diferentes tipos e coberturas de vegetação, incluindo região urbana, registraram maiores riquezas de espécies dessas famílias, 26 e 15, respectivamente. Muitas espécies de Papilionidae são bons indicadores de matas bem conservadas e de recursos hídricos abundantes (Brown JR \& Freitas 1999). Nas espécies pertencentes à tribo Troidini, as larvas alimentam-se exclusivamente de Aristolochiaceae (TYLER et al. 1994), incluindo Aristolochia triangularis, bastante comum nos locais amostrados no presente trabalho.

Dos três locais estudados, $M$ apresentou a maior riqueza ( $\mathrm{S}=103$ ) e abundância de indivíduos ( $\mathrm{N}=602$ ) (Tab. I e III). A menor riqueza foi observada em $\mathrm{E}(\mathrm{S}=79)$ e a menor abundância em C ( $\mathrm{N}=419)$ (Tab. I e III). Conforme a figura 2, o número de espécies observado está quase atingindo o esperado.

Em relação à distribuição de percentagem de riqueza de

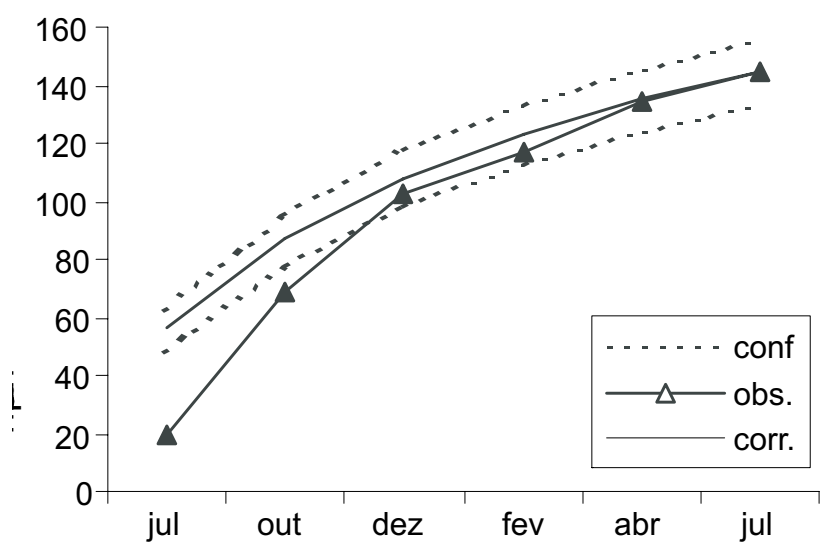

Figura 2. Curva de suficiência amostral, obtida em seis ocasiões amostrais, no Morro do Elefante, Morro do Lar Metodista e Morro Cerrito, Santa Maria, Rio Grande do Sul, entre julho de 2004 e julho de 2005. (conf.) Intervalo de confiança, (obs) número acumulado de espécies observado, (corr.) número acumulado de espécies corrigido.

espécies por família, M e C apresentaram a mesma ordenação (da maior para a menor representatividade): Nymphalidae, Hesperiidae, Lycaenidae, Papilionidae e Pieridae, enquanto em E Lycaenidae foi superior à de Papilionidae (Fig. 3). Quanto a abundância percentual de indivíduos por família, E e M foram semelhantes: Nymphalidae (com a maior representatividade), seguida por Hesperiidae, Papilionidae, Lycaenidae e Pieridae, enquanto em C Pieridae foi superior a Lycaenidae (Fig. 4).

As espécies mais abundantes nos locais estudados foram os ninfalídeos Dryas iulia alcionea (Cramer, 1779) ( $n=146)$, Heliconius erato phyllis $(n=121)$ (Fabricius, 1775) e Paryphthimoides phronius (Godart, [1824]) ( $n=90)$ e o hesperídeo Pyrgus orcus (Stoll, 1780) ( $n=90)$ (Tab. I). Essa composição mantém-se quase a mesma analisando-se cada um dos locais separadamente. A espécie mais abundante em $\mathrm{E}$ foi Heliconius erato phyllis ( $\mathrm{n}$ $=55$ ), em M e C, Dryas iulia alcionea ( $n=58$ e $n=36$, respectivamente) (Tab. I). A maioria dessas espécies possui ampla distribuição, sendo comuns em vários habitats e características de locais semi-abertos ou perturbados (BROWn JR 1992, MARCHIORI \& RomANowskı 2006), como observado em alguns trechos dos locais estudados.

Revista Brasileira de Zoologia 24 (1): 108-120, março 2007 

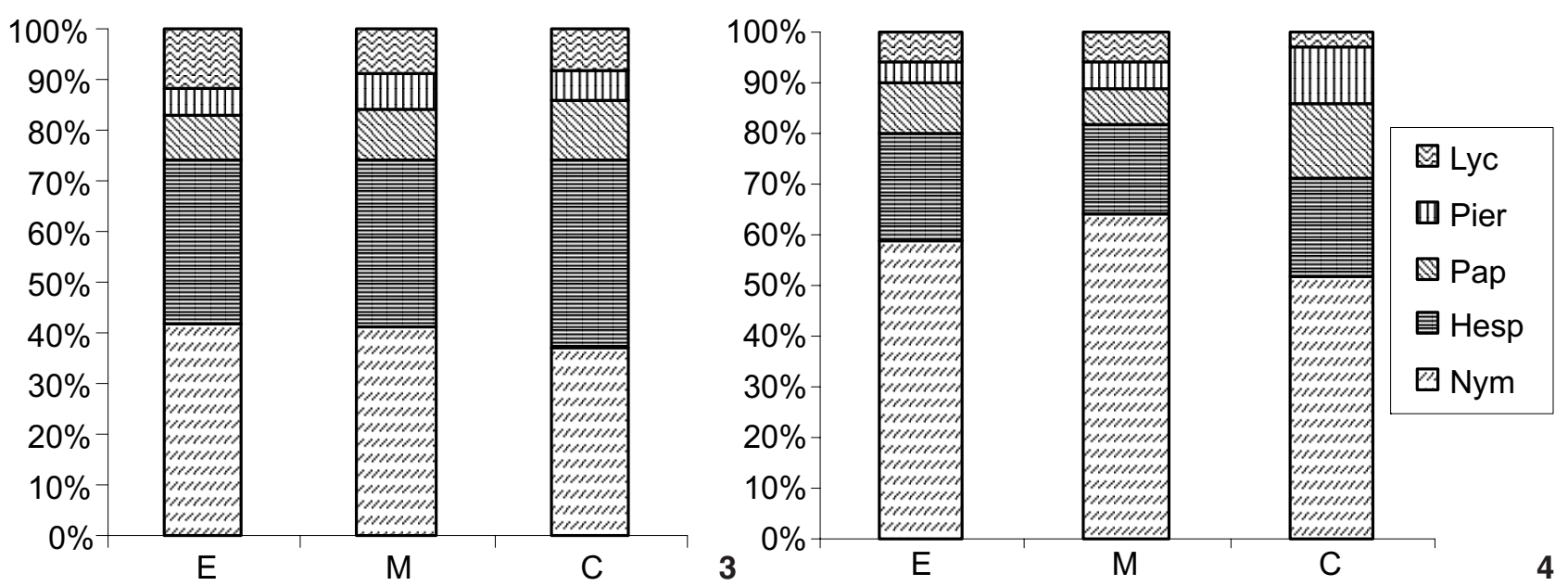

Figuras 3-4. (3) Riqueza de espécies (\%) e (4) abundância (\%) por família de borboletas registradas no Morro do Elefante (E), Morro do Lar Metodista (M) e Morro Cerrito (C), Santa Maria, Rio Grande do Sul, entre julho de 2004 e julho de 2005. (Nym) Nymphalidae, (Hesp) Hesperiidae, (Pap) Papilionidae, (Pier) Pieridae, (Lyc) Lycaenidae.

\section{Diversidade, Dominância e Similaridade}

Os índices de diversidade de Shannon-Wiener e de Margal ef calculados tiveram a mesma ordenação nos três locais (Tab. III). Os maiores valores foram registrados em M, já mencionado como o local com maior riqueza e abundância de indivíduos, demonstrando com isto melhor distribuição dos indivíduos entre as espécies (equitatividade). Além disso, M possui a maior heterogeneidade estrutural dos três locais amostrados, com distintas formações vegetacionais, fator favorável ao aumento da diversidade de borboletas, segundo BAZ \& BOYERO (1995). Porém, este é o local mais perturbado antropicamente, com presença de algumas ruínas e jardins de antigas moradias e trânsito mais ou menos regular de pessoas que vivem nas proximidades. No entanto, paisagens perturbadas também podem prover condições adequadas para permanência e maior abundância de algumas espécies, principalmente boas colonizadoras (Kocher \& WILLIANs 2000). C, com valores intermediários de diversidade, possui a menor área e encontra-se isolado, delimitado por rodovias e a zona urbana, porém oferece grande quantidade de recursos às borboletas. Os menores valores de diversidade ocorreram em E, local com a menor riqueza de espécies mas que possui a maior cobertura de vegetação arbórea, contígua com a escarpa do Planalto e mais afastado da região urbana, sendo ainda o menos perturbado por trânsito de pessoas dos três locais estudados.

Os índices de dominância de Simpson e de Berger-Parker foram mais representativos em E e menos em C (Tab. III), corroborando com o maior número de espécies mais abundantes encontradas naquele local (Fig. 5), incluindo a única espécie considerada dominante ( $\mathrm{H}$. erato; $\mathrm{fr}=0,101)$.

Do total de espécies, 43 (30\%) foram comuns aos três locais (Tab. I): 21 Nymphalidae, 10 Hesperiidae, seis Papilionidae,
Tabela III. Número de espécies (S), número de indivíduos (N), índice de Diversidade de Shannon-Wiener $\left(\mathrm{H}^{\prime}\right)$ e de Margalef (Dmg), índice de Dominância de Simpson (D) e de Berger-Parker (d), número de espécies registradas exclusivamente em um dos locais (Excl.), número destas espécies representadas por mais de um indivíduo (Excl. $>1$ ) e número de espécies representadas por um único indivíduo (Singleton), registradas no Morro do Elefante (E), Morro do Lar Metodista (M) e Morro Cerrito (C), Santa Maria, Rio Grande do Sul, entre julho de 2004 e julho de 2005.

\begin{tabular}{lcccc}
\hline & $\mathrm{E}$ & $\mathrm{M}$ & $\mathrm{C}$ & Total \\
\hline $\mathrm{S}$ & 79 & 103 & 89 & 145 \\
$\mathrm{~N}$ & 543 & 602 & 419 & 1564 \\
$\mathrm{H}^{\prime}$ & 3,605 & 3,928 & 3,868 & 4,066 \\
$\mathrm{Dmg}$ & 12,39 & 16,09 & 14,57 & 19,71 \\
$\mathrm{D}$ & 0,0456 & 0,0343 & 0,0332 & 0,0317 \\
$\mathrm{~d}$ & 0,1013 & 0,0964 & 0,0859 & 0,0934 \\
Excl. & 11 & 25 & 25 & 61 \\
Excl. $>1$ & 7 & 9 & 10 & 26 \\
Singleton & 4 & 17 & 16 & 37 \\
\hline
\end{tabular}

quatro Lycaenidae e dois Pieridae, a maioria delas freqüentes em ambientes abertos ou perturbados (Brown JR 1992).

Cerca da metade (61) das espécies registradas foram exclusivas de um dos locais: 25 em C e em M, a maioria pertencendo à família Hesperiidae no primeiro, e Hesperiidae e Nymphalidae no segundo; e 11 em E, a maioria Hesperiidae (Tab. I e III). Não foi possível diferenciar os três locais pela sua composição de espécies exclusivas, pois todos possuem espécies associadas a ambientes perturbados, abertos ou clareiras e al gumas associadas a ambientes florestais (Brown JR 1992), e faltam informações sobre a biologia de muitas delas. Mas é interessante observar o 

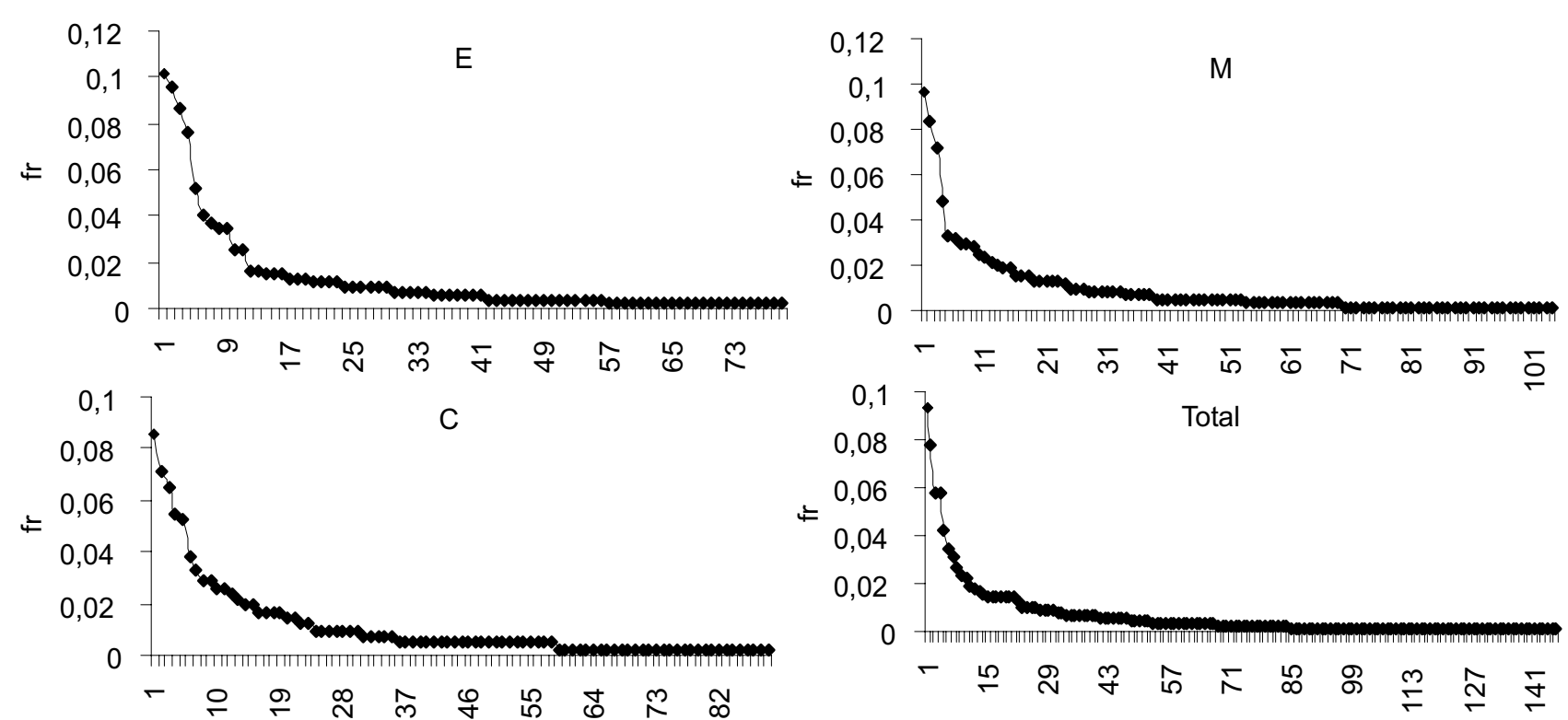

Figura 5. Distribuição das freqüências relativas das espécies de borboletas, no Morro do Elefante (E), Morro do Lar Metodista (M) e Morro Cerrito (C), e em todos locais (Total), Santa Maria, Rio Grande do Sul, entre julho de 2004 e julho de 2005. fr: freqüência relativa.

maior valor em C, com menor área, mas rico em recursos como néctar, frutos e barro, além da heterogeneidade de ambientes. Ao considerar somente as espécies exclusivas representadas por mais de um indivíduo em cada local, C e M mantiveram os maiores valores (10 e nove, respectivamente), enquanto que $E$ teve sete espécies (com representatividade maior, 64\%, em relação ao número de espécies exclusivas representadas por um indivíduo nesse mesmo local) (Tab. III). Sete espécies exclusivas estão entre os novos registros para o Rio Grande do Sul, sendo que quatro ocorreram em C, dois em $\mathrm{E}$ e apenas um em M (Tab. I).

Das espécies registradas em Santa Maria, 37 (26\%) foram representadas por "singletons", destas, 17 ocorreram em M, 16 em C e quatro em E (Tab. I e III). Hesperiidae foi a família mais numerosa entre os singletons, com 20 espécies (54\%), seguida de Nymphalidae (8 - 22\%), Lycaenidae (7 - 19\%) e Papilionidae (2 - 5\%). Estas espécies equivaleram a $18 \%$ do total de espécies amostradas em C, 16\% em M e 5\% em E. Dos "singletons" identificados, seis também são novas espécies registradas para o Estado, três em C, duas em E e uma em M (Tab. I). Muitos "singletons" são espécies que residem no habitat em que são amostradas e podem ser muito difíceis de encontrar, em qualquer lugar ou época, uma vez que mantêm-se em populações pequenas, sazonais e erráticas (Brown Jr \& Freitas 1999, Novotný $\&$ BASSET 2000). Isso pode estar acontecendo em Santa Maria, uma vez que a maioria dos "singletons" encontrados pertencem às famílias Hesperiidae e Lycaenidae, reconhecidamente de difícil amostragem. Além disso, 17 "singletons" (12 Hesperiidae, quatro Nymphalidae e um Lycaenidae) também são considerados raros na literatura consultada (BIEZANKo 1963, BieZANKo \& Mielke 1973, Biezanko et al. 1978, Brown Jr 1992, Mielke \&
Casagrande 1997) (Tab. I). Desses, oito foram registrados em C seteem $M$ edois em $E$, sendo quetrês, Milanion leucaspis (Mabille, 1878), Lychnuchoides ozias ozias (Hewitson, 1878) e Synapte silius (Latreille, [1824]), são considerados habitantes de florestas (BRown JR 1992). Existe sempre um certo número de espécies raras nas comunidades, no entanto, o conceito de rara varia conforme o autor (MAGURRAN 2004), assim como também de acordo com as características locais. Como exemplo, no presente trabalho, pelo menos uma espécie "singleton" considerada rara na literatura, Ortilia ithra (Kirby, 1900), foi registrada dentre as mais abundantes em ambiente de restinga, no Parque Estadual de Itapuã, Rio Grande do Sul (MARCHIORI \& ROMANOWSKI 2006). Enquanto outra espécie, Panoquina ocola (W.H. Edwards, 1863), considerada rara, teve aqui o registro de 15 indivíduos. Por isso, no presente trabalho está sendo considerado "singleton" como sinônimo de raro, conforme proposto por NovotnÝ \& BASSET (2000). Enfatizouse apenas as espécies também consideradas raras na literatura consultada, para evitar artifícios relacionados a um esforço amostral ainda pequeno. Deve-se estimular e ampliar a realização de mais inventários de borboletas no Rio Grande do Sul, com metodologias padronizadas para comparação e confirmação dos dados obtidos até o momento.

O maior valor para o índice de Morisita-Horn foi apresentado por E e M (Figs 6 e 7). O maior número de espécies abundantes compartilhadas por ambos locais tem peso considerável nesse índice de similaridade, cujo menor valor de similaridade correspondeu a E e C. O índice de Jaccard seguiu a mesma ordenação, maior entre E e $M(0,525)$ e a menor entre $E$ e C $(0,4)$, mostrando que os Morros do Elefante e do Lar Metodista possuem maior semelhança na sua composição de

Revista Brasileira de Zoologia 24 (1): 108-120, março 2007 

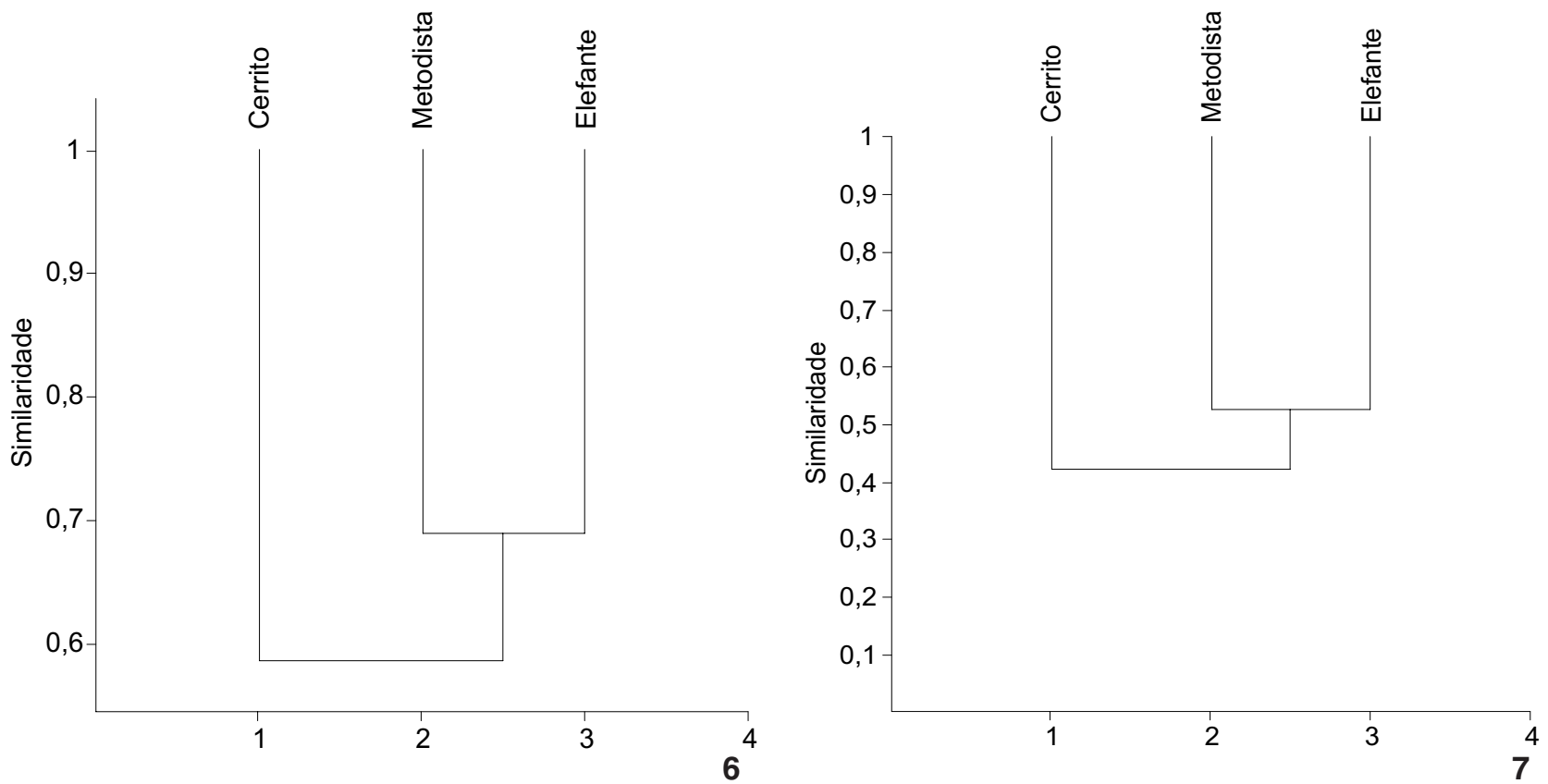

Figuras 6-7. (6) Similaridade de Morisita-Horn e de (7) Jaccard entre o Morro do Elefante, Morro do Lar M etodista e Morro Cerrito, Santa Maria, Rio Grande do Sul, entre julho de 2004 e julho de 2005.

espécies, comparados ao Cerrito. Tais dados condizem com a condição isolada do Morro Cerrito na Depressão Central, em relação aos outros dois locais estudados, localizados na escarpa do Planalto.

Concluindo, a fauna de borboletas dos fragmentos estudados da Floresta Estacional Decidual de Santa Maria apresenta-se bastante rica e composta por al gumas espécies indicadoras de boas condições ambientais, apesar da crescente devastação de suas áreas naturais. Essa fauna possui composição mais próxima de outras comunidades amostradas no Estado do Rio Grande do Sul, contrastando com o restante do país, apresentando uma tendência à diminuição de representatividade da família Lycaenidae em prol do aumento das famílias Papilionidae e Pieridae.

Nesta pesquisa, foram registradas nove espécies de borboletas ainda não referidas para o Rio Grande do Sul. Os três locais amostrados, apesar de diferenças em área, isolamento, fisionomia e impacto antrópico, apresentaram semelhanças aproximadas de 50 a $70 \%$ em sua composição de espécies. No entanto, eles também apresentaram uma proporção de aproximadamente $42 \%$ de espécies exclusivas, representativas de cada local. Sugere-se o aumento do número de horas de amostragem para confirmação e ampliação dos resultados encontrados. Porém, mesmo depois de um inventário relativamente breve, como o efetuado no presente trabalho, já existem subsídios para enfatizar a importância da conservação e preservação desses habitats remanescentes da Floresta Estacional Decidual de Santa Maria e sua biodiversidade.

\section{AGRADECIMENTOS}

Aos responsáveis pelas Instituições situadas nos locais de pesquisa: Cidade dos Meninos, Lar M etodista e Centro Marista de Eventos. A Gabriel D. Sackis (UFSM) por auxílio no campo; À Ana L. Paz, Maria O. Marchiori, Cristiano A. Iserhard e Fabiana de Camargo (UFRGS) pela ajuda nas identificações. A Curtis J. Callaghan e Olaf H.H. Mielke (UFPR), Ronaldo B. Francini (Unisantos) e Robert K. Robbins (Smithsonian Institution) pelas valiosas identificações de Lycaenidae (Riodininae), Hespe riidae, Nymphalidae (Actinote) e Lycaenidae (Theclinae). À Helena P. Romanowski (UFRGS) e dois revisores anônimos pelas sugestões rel evantes e revisão crítica do trabal ho. À CAPES pela concessão de bolsa de estudos.

\section{REFERÊNCIAS BIBLIOGRÁFICAS}

Alberti, L.F.; J.A.N. HiRT; D.B.F. Machado JR; M. Steckel \& C.S. Tombonı. 2000. Aspectos florísticos e síndromes de dispersão das espécies arbóreas do Morro de Santo Antão, Santa Maria, RS. Revista Ciência e Natura 22: 145-160.

BAZ, A. \& A.G. BOYERO. 1995. The effects of forest fragmentation on butterfly communities in central Spain. Journal of Biogeography 22: 129-140.

Beccaloni, G.W. \& K.J. Gaston. 1995. Predicting species richness of Neotropical forest butterflies: Ithomiinae (Lepidoptera: Nymphalidae) as indicators. Biological Conservation 71: 77-86.

BiezAnKo, C.M. 1949. Acraeidae, Heliconidae e Nymphalidae 
de Pelotas e seus arredores. Pelotas, Livraria do Globo, 16p. BiezAnko, C.M. 1958. Ib. Pieridae da Zona Sueste do Rio Grande do Sul. Arquivos de Entomologia, Série A: 1-15.

Biezanko, C.M. 1959a. Ia. Papilionidae da Zona Sueste do Rio Grande do Sul. Arquivos de Entomologia, Série A: 1-17.

BiezAnko, C.M. 1959b. Ia. Papilionidae da Zona Missioneira. Arquivos de Entomologia, Série B: 1-12.

BiezAnko, C.M. 1960a. Ib. Pieridae da Zona Missioneira do Rio Grande do Sul. Arquivos de Entomologia, Série B: 1-12.

BieZANKo, C.M. 1960b. III. Danaidae et Ithomidae da Zona Sueste do Rio Grande do Sul. Arquivos de Entomologia, Série $A:$ : 1-6.

Biezanko, C.M. 1960c. III. Danaidae et Ithomidae da Zona Missioneira do Rio Grande do Sul. Arquivos de Entomologia, Série B: 1-6.

BiezAnKo, C.M. 1960d. IV. Satyridae, Morphidae et Brassolidae da Zona Sueste do Rio Grande do Sul. Arquivos de Entomologia, Série A: 1-13.

Biezanko, C.M. 1960e. IV. Satyridae, Morphidae et Brassolidae da Zona Missioneira do Rio Grande do Sul. Arquivos de Entomologia, Série B: 1-10.

BiezAnko, C.M. 1963. VI. Hesperiidae da Zona Sueste do Rio Grande do Sul. Arquivos de Entomologia, Série A: 1-25.

Biezanko, C.M. \& R.G. Freitas.1938. Catálogo dos insetos encontrados na cidade de Pelotas e seus arredores. 1. Lepidópteros. Pelotas, Escola de Agronomia Eliseu Maciel, 32p.

BiezAnko, C.M. \& O.H. Mielke. 1973. Contribuição ao estudo faunístico dos Hesperiidae americanos. IV Espécies do Rio Grande do Sul, Brasil, com notas taxonômicas e descrições de espécies novas (Lepidoptera). Acta Biológica Paranaense 2 (1-4): 51-102.

Biezanko, C.M.; O.H. Mielke \& A. Wedderhoff. 1978. Contribuição ao estudo faunístico dos Riodinidae do Rio Grande do Sul, Brasil (Lepidoptera). Acta Biológica Paranaense 7 (14): 7-22.

BogGS, C.L.; W.B. W ATT \& P.R. EHRLICH. 2003. Butterflies: ecology and evolution taking flight. Chicago, The University of Chicago Press, 739p.

BRown JR, K.S. 1992. Borboletas da Serra do Japi: diversidade, habitats, recursos alimentares e variação temporal, p. 142186. In: L.P.C. MoRellato, (Ed.). História natural da Serra do Japi: ecologia e preservação de uma área florestal no sudeste do Brasil. Campinas, Editora da Unicamp, 321p.

Brown JR, K.S. 1996. Diversity of Brazilian Lepidoptera: history of study, methods for measurement, and use as indicator for genetic, specific and system richness, p 221-253. In: C.E.M. Bicudo \& N.A. MENEZES (Eds). Biodiversity in Brazil: a first approach. São Paulo, Instituto de Botânica, CNPq, 326p.

Brown JR, K.S. \& A.V.L. FreitAs. 1999. Lepidoptera, p. 227-243. In: C.R.F. Brandão \& E.M. Cancello (Eds). Biodiversidade do Estado de São Paulo, Brasil. Invertebrados terrestres. São Paulo, FAPESP, XVI+279p.

Brown JR, K.S. \& A.V.L. FreitAs. 2002. Butterfly communities of urban forest fragments in Campinas, São Paulo, Brazil: structure, instability, environmental correlates, and conservation. Journal of Insect Conservation 6 (4): 217-231.

Brown JR, K.S. \& R.W. Hutchings. 1997. Disturbance, fragmentation, and the dynamics of diversity in Amazonian forest butterflies, p. 91-110. In: W.F. LAURENCE \& R.O. BierRegaARD JR (Eds). Tropical forest remnants: ecology, mangement and conservation of fragmented communities. Chicago, University of Chicago Press.

Canals, G.R. 2000. Butterflies of Buenos Aires. Buenos Aires, L.O.L.A., 347p.

Canals, G.R. 2003. Mariposas de Misiones. Buenos Aires, L.O.L.A., 492p.

ColweLL, R.K. 2005. Estimates: statistic estimation of species richness and shared species from samples. Version 7.5. Disponível na World Wide Web em: http://viceroy.eeb. uconn.edu/Estimates [acesso em 07.I.2006].

Corseuil, E.; F.C. Quadros; J.A. Teston \& A. Moser. 2004. Borboletas (Lepidoptera, Papilionoidea e Hesperioidea) coletadas no Centro de Pesquisas e Conservação da Natureza Pró-Mata. 4. Lycaenidae. Divulgação do Museu de Ciência e Tecnologia 9: 65-70.

D'ABRerA, B. 1988. Butterflies of the Neotropical Region. Part V. Nymphalidae (conc.) \& Satyridae. Victoria, Hill House, IX+p. 680-877.

D'ABRERA, B. 1994. Butterflies of the Neotropical Region. Part VI. Riodinidae. Victoria, Hill House, IX+p. 880-1096.

D'ABRERA, B. 1995. Butterflies of the Neotropical Region. Part VII. Lycaenidae. Victoria, Hill House, XI+p.1098-1270.

Emery, E.O.; K.S. Brown Jr \& C.E.G. Pinheiro. 2006. As borboletas (Lepidoptera, Papilionoidea) do Distrito Federal, Brasil. Revista Brasileira de Entomologia 50 (1): 85-92.

FreitAS, A.V.L \& K.S. Brown JR. 2004. Phylogeny of the Nymphalidae (Lepidoptera). Systematic Biology 53 (3): 1-25.

HePpNer, J.B. 1991. Faunal regions and the diversity of Lepidoptera. Tropical Lepidoptera 2 (1): 1-85.

ISERHARD, C.A.A. \& H.P. RomanowSKI. 2004. Lista de espécies de borboletas (Lepidoptera, Papilionoidea e Hesperioidea) da região do rio Maquiné, Rio Grande do Sul, Brasil. Revista Brasileira de Zoologia 21 (3): 649-662.

KoCHER, S.D. \& E.H. WiLliAms. 2000. The diversity and abundance of North American butterflies vary with habitat disturbance and geography. Journal of Biogeography 27: 785-794.

KRüger, C.P. \& E.J.E. SI lva. 2003. Papilionoidea (Lepidoptera) de Pelotas e seus arredores, Rio Grande do Sul, Brasil. Entomología y Vectores 10 (1): 31-45.

LAMAS, G. 2004. Checklist: Part 4A. Hesperioidea- Papilionoidea. In: J.B. Heppner (Ed.). Atlas of Neotropical Lepidoptera 5A. Gainesville, Association for Tropical Lepidoptera, XXXVI+ 439p.

Link, D.; C.M. Biezanko; M.F. Tarragó \& S. Carvalho. 1977. Lepidoptera de Santa Maria e arredores. I: Papilionidae e Pieridae. Revista do Centro Ciências Rurais 7 (4): 381-389. 
Machado, P.F.S. \& S.J. Longhi. 1990. Aspectos florísticos e fitossociológicos do "Morro do Elefante", Santa Maria, RS. Revista do Centro de Ciências Rurais 20 (3/4): 261-280.

MagurRan, A.E. 2004. Measuring biological diversity. Oxford, Blackwell Publishing Company, 256p.

MARCHIORI, M.O. \& H.P. RomANOWSKI. 2006. Species composition and diel variation of a butterfly taxocenose (Lepidoptera, Papilionoidea e Hesperioidea) in a restinga forest at Itapuã State Park, Rio Grande do Sul, Brazil. Revista Brasileira de Zoologia 23 (2): 443-454.

MielKe, O.H. 1979. Contribuição ao estudo faunístico dos Hesperiidae americanos. V. Nota suplementar às espécies de PyrrhopyginaeePyrginae do Rio Grande do Sul, Brasil (Lepidoptera). Acta Biológica Paranaense 8/9: 7-17. [1980].

MieLKE, O.H. 2005.Catalogue of the American Hesperioidea: Hesperiidae (Lepidoptera). Volume 1. Complementary and supplementary parts to the checklist of the Neotropical region. Hesperioidea: Hesperiidae: Pyrrhopyginae. Curitiba, Sociedade Brasileira de Zoologia, vol. 1, 125p.

Mielke, O.H. \& M.M. Casagrande. 1997. Papilionoidea e Hesperioidea (Lepidoptera) do Parque Estadual do Morro do Diabo, Teodoro Sampaio, São Paulo, Brasil. Revista Brasileira de Zoologia 14 (4): 967-1001.

MoReno, C.E. 2001. Métodos para medir la biodiversidad. Zaragova, Cited/Unesco \& SEA, Manuales y Tesis SEA, 84p.

MotтA, P.C. 2002. Butterflies from the Uberlândia region, central Brazil: species list and biological comments. Brazilian Journal of Biology 62 (1): 151-163.

NEw, T.R. 1997. Are Lepidoptera an effective 'umbrela group' for biodiversity conservation? Journal of Insect Conservation 1: 5-12.

New, T.R.; R.M. Pyle; J.A. Thomas \& P.C. Hammond. 1995. Butterfly Conservation Management. Annual Review of Entomology 40: $57-83$.

Nimer, E. 1990. Clima, p. 151-187. In: Fundação Instituto BrasileiRo de Geografia e Estatística (Ed). Geografia do Brasil: Região Sul. Rio de Janeiro, SERGRAF/ IBGE, vol. 2, 420p.

NovotnÝ, V. \& Y. BASSET. 2000. Rare species in communities of tropical insect herbivores: pondering the mistery of singletons. Oikos 89: 564-572.

Pereira, P.R.B.; L.R. Garcia Netto; C.J.A. Borin \& M.G.B. Sartori. 1989. Contribuição à geografia física do município de Santa Maria: unidades de paisagem. Geografia, Ensino e Pesquisa 3: $37-68$.

Recebido em 06.VI.2006; aceito em 05.III.2007.
PolLARD, E. 1977.A method for assessing changes in the abundance of butterflies. Biological Conservation 12: 115-134.

Quadros, F.C.; A.L. Dorneles \& E. CorseuIL. 2004. Ninfalídeos (Lepidoptera, Nymphalidae) ocorrentes no Norte da Planície Costeira do Rio Grande do Sul, Brasil. Biociências 12 (2): 147164.

Robaina, L.E.; M.G. Berger; S.S.V. Cristo \& P.M. Paula. 2001. Análise dos ambientes urbanos de risco do município de Santa Maria - RS. Revista Ciência e Natura 23: 127-137.

SchwARTZ, G. \& R.A. Di MARE. 2001. Diversidade de quinze espécies de borboletas (Lepidoptera: Papilionidae) em sete comunidades de Santa Maria, RS. Ciência Rural 3: 49-55.

Teston, J.A. \& E. CorseuIL. 1998. Lista documentada dos Papilionídeos (Lepidoptera, Papilionidae) do Rio Grande do Sul, Brasil. Biociências 6 (2): 81-94.

Teston, J.A. \& E. CorseuIL. 1999. Borboletas (Lepidoptera, Rhopalocera) ocorrentes no Centro de Pesquisas e Conservação da Natureza Pró-Mata. 1. Papilionidae. Divulgação do Museu de Ciência e Tecnologia 4: 217-228.

Teston, J.A. \& E. Corseuil. 2000a. Borboletas (Lepidoptera, Rhopalocera) ocorrentes no Centro de Pesquisas e Conservação da Natureza Pró-Mata. 2. Pieridae. Divulgação do Museu de Ciência e Tecnologia 5: 143-155.

Teston, J.A. \& E. Corseuil. 2000b. Lista documentada dos Pierídeos (Lepidoptera, Pieridae) do Rio Grande do Sul, Brasil. Biociências 8 (2): 115-132.

Teston, J.A. \& E. Corseuil. 2001. Ninfalídeos (Lepidoptera, Nymphalidae) ocorrentes no Rio Grande do Sul, Brasil. Parte I. Danainae e Ithomiinae. Biociências 9 (1): 51-61.

Teston, J.A. \& E. Corseuil. 2002a. Borboletas (Lepidoptera, Rhopalocera) ocorrentes no Centro de Pesquisas e Conservação da Natureza Pró-Mata. 3. Nymphalidae. Divulgação do Museu de Ciência e Tecnologia 7: 1-208.

Teston, J.A. \& E. Corseult. 2002b. Ninfalídeos (Lepidoptera, Nymphalidae) ocorrentes no Rio Grande do Sul, Brasil. Parte II. Brassolinae e Morphinae. Biociências 10 (1): 75-84.

Tumuhimbise, G.; M.J.N. Okwakol \& T.N. Kangwagye. 2001. Species diversity of swall owtail butterflies (Papilionidae: Lepidoptera) in North Maramagambo Forest. African Journal Ecology 39 (1): 113-115.

TYLER, H.A.; K.S. BROWn JR \& K.H. WILSON. 1994. Swall lowtail butterflies of the Americas: a study in biological dynamics, ecological diversity, biosystematics, and conservation. Gainesville, Scientific Publishers, 376p. 